\title{
Nuclear artifacts in gastric endoscopic submucosal dissection specimens: A clinicopathological study
}

\author{
SUSUMU MATSUKUMA ${ }^{1,2}$, HIROAKI TAKEO ${ }^{2}$ and KIMIYA SATO ${ }^{2}$ \\ ${ }^{1}$ Health Care Center and ${ }^{2}$ Department of Pathology, Japan Self-Defense Forces Central Hospital, Tokyo 154-0001, Japan
}

Received May 8, 2014; Accepted May 29, 2014

DOI: $10.3892 / \mathrm{mco} .2014 .322$

\begin{abstract}
To delineate the characteristics of nuclear artifacts associated with endoscopic submucosal dissection (ESD), we examined 97 gastric ESD specimens from 79 patients. In 69 of the specimens (71\%), multinucleated figures and/or atypical mitotic-like figures, including tripolar-like and bizarre spindles, were found in the peripheral portions close to the marking areas. These nuclear figures were mostly recognizable as artifacts, but were infrequently (13 specimens) accompanied by other nuclear alterations and/or architectural abnormalities, mimicking dysplasia. However, in the deep cut sections, the dysplastic characteristics tended to disappear and coagulative or degenerative findings became more prominent. These nuclear artifacts were not found in 69 age- and gender-matched control gastrectomy specimens without ESD. Multinucleated artifacts were associated with the size of the ESD specimens $(\mathrm{P}=0.003)$, frequency of marking $(\mathrm{P}<0.001)$ and a history of 'previous' marking 1-6 days prior to ESD $(\mathrm{P}<0.001)$; however, they were not associated with age, ESD procedure time, or 'fresh' marking on the day of the ESD. Atypical mitosis-like characteristics were associated with a history of 'fresh' $(\mathrm{P}=0.007)$ as well as 'previous' $(\mathrm{P}=0.002)$ marking, but not with other variables. Dysplasia-like artifacts were associated with older age only $(\mathrm{P}=0.031)$. Follow-up data of all the patients with nuclear artifacts showed no aggressive behavior. Therefore, we concluded that these nuclear changes were ESD-related artifacts. Particularly in older patients, these changes may simulate dysplasia and must be distinguished from true dysplasia or neoplasia.
\end{abstract}

\section{Introduction}

Since the early 1980 s, endoscopic resection (ER) techniques using electrothermal devices, such as polypectomy, strip biopsy and endoscopic mucosal resection (EMR), have been devel-

Correspondence to: Dr Susumu Matsukuma, Health Care Center, Japan Self-Defense Forces Central Hospital, Ikejiri 1-2-24, Setagaya-ku, Tokyo 154-0001, Japan

E-mail: skuma@cocoa.plala.or.jp

Key words: stomach, endoscopic submucosal dissection, artifact, multinucleated cells, atypical mitosis, electrothermal injury, cautery oped as diagnostic and curative procedures for gastrointestinal mucosal lesions (1-6). The latest modality using specialized electrosurgical knives, referred to as endoscopic submucosal dissection (ESD), has enabled more complete removal of gastric lesions and is currently accepted as a standard treatment of early gastric adenocarcinoma (EGA) (6-8). The precise histological assessment of ER specimens, such as pathological diagnosis, the depth of cancerous invasion, lymphovascular permeation and the status of the surgical margin, is crucial for the subsequent management of the patients $(1,3,4,6-9)$. Therapeutic procedures using electrothermal devices may induce varying artifacts disturbing the histological evaluation in gastrointestinal and other specimens (1,3,4,9-14). However, histological artifacts in newly developed ESD specimens remain poorly understood. In this study, we histologically examined ESD specimens focusing on artifactual nuclear changes and evaluated their association with clinical findings.

\section{Materials and methods}

Materials. A total of 97 gastric ESD specimens from 79 patients were retrieved from the surgical pathology files of the Department of Pathology, Japan Self-Defense Forces Central Hospital, Tokyo, Japan (2009-2012). Clinical information was available from the patients' charts. If necessary, the gross photographs of the ESD specimens were also reviewed. A total of 18 patients had metachronously undergone ESD (17, twice; 1 , three times) and the final diagnoses included 74 patients with adenocarcinoma (73 patients with EGA and 1 with advanced cancer), 14 with tubular adenoma, 3 with carcinoid tumor and 6 with hyperplastic polyp of the foveolar type. Using an electrical device, preoperative marking was placed outside the gastric lesions in 95 specimens, 0-6 days prior to ESD (mean, 1.74 days), once in 37 and twice in 58 specimens. In this study, we defined marking 1-6 days prior to ESD as 'previous marking' and marking on the same day as 'fresh marking'; the former was performed in 76 specimens using an argon plasma coagulator (APC; ERBE Elektromedizin $\mathrm{GmbH}$, Tübingen, Germany) and the latter was performed from 10 min to a few hours prior to ESD. An APC was used in 69, flush knife in 5 and precutting knife in 2 specimens. The 95 specimens with marking were divided into 19 with fresh marking only, 19 with previous marking only (including 1 with previous marking twice) and 57 with both fresh and previous marking. The dissecting device was an insulation-tipped (IT) 

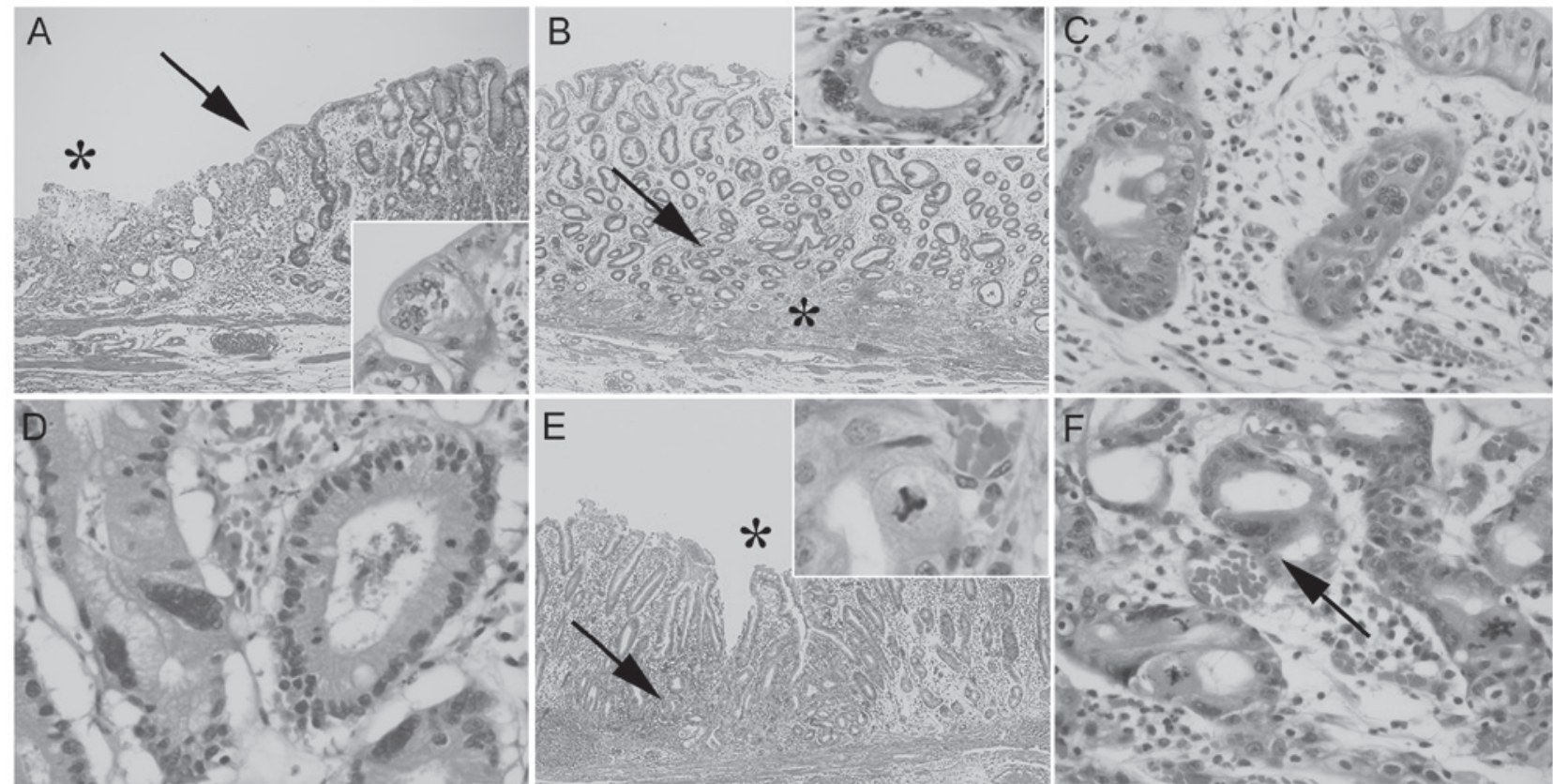

Figure 1. Endoscopic submucosal dissection-related nuclear artifacts. (A) Surface changes (arrow) near the eroded marking point (asterisk) and a high-power view (inset) showing multinucleated cells. (B) Minute changes (arrow) in the deep mucosa near the coagulated area (asterisk) and a high-power view (inset) showing multinucleation. (C) Scattered multinucleation in the middle mucosal portion. (D) Conjugated multinucleation simulating gigantic nuclei. (E) Minute change (arrow) beneath eroded marking point (asterisk) and a high-power view (inset) showing tripolar-like nuclei. (F) Scattered bizarre nuclei and multinucleation (arrow)
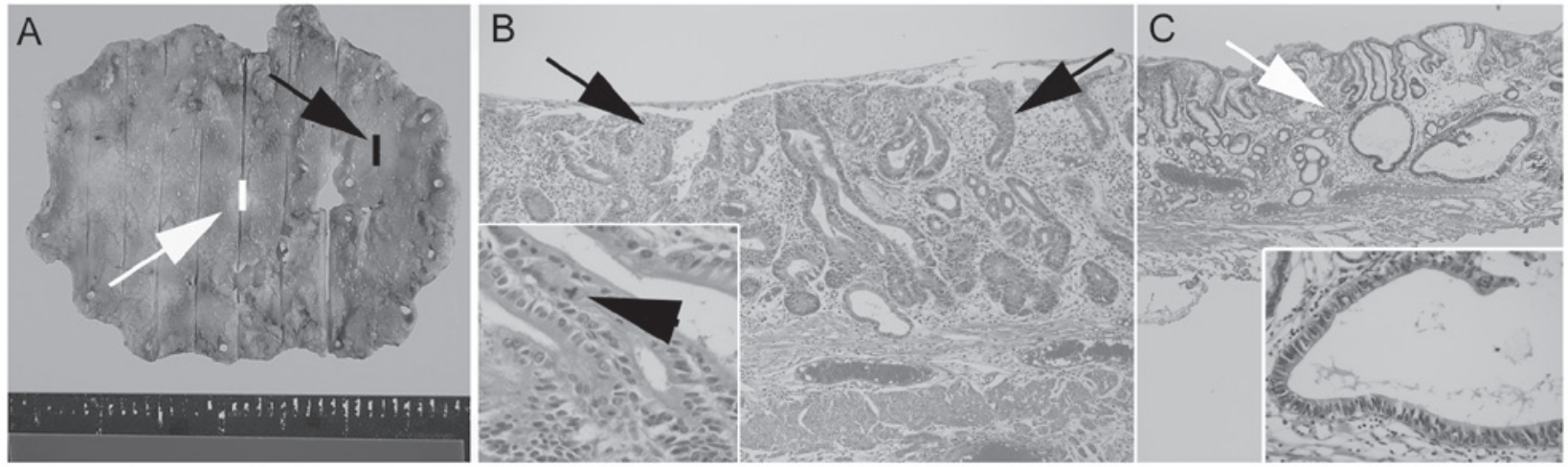

Figure 2. A case of endoscopic submucosal dissection (ESD)-related artifact misdiagnosed as cancer. (A) Peripherally located dysplasia-like artifact (black line and arrow) near marking points and centrally situated adenocarcinoma (white line and arrow) in ESD specimens. (B) Aggregated dysplasia-like glands (arrows) and a high-power view (inset) showing cribriform-like structures and hyperchromatic, disarranged nuclei, with focal tripolar-like nucleus (arrowhead). (C) Degenerated tubules (white arrow) in the central portion of the ESD specimen (white line, Fig. 2A) and a high-power view (inset) showing hyperchromatic, disarranged and swollen cancerous nuclei.

knife (Olympus, Tokyo, Japan) with or without other devices (precutting, flex and/or hook knives) in 82, flush knife only in 14 and flex knife only in 1. Diathermic hemostasis using Coagrasper $^{\mathrm{TM}}$ (Olympus) was performed in 90 specimens. The procedure time of ESD ranged between 7 and 470 min (mean, $91.4 \mathrm{~min})$. For a control study, 79 gastrectomy specimens from patients with gastric cancer who had no history of ESD were retrieved from the surgical pathology files of the Department of Pathology, Japan Self-Defense Forces Central Hospital (1997-2008) and examined. The number of sections examined in each control case ranged between 8 and 105 (mean, 22.3).

Methods. The ESD specimens were fixed in $15 \%$ buffered formalin for $14-52 \mathrm{~h}$ and were completely cut at 3-4-mm inter- vals, ranging in each case between 4 and 45 sections (mean, 13.4 sections). The sections $(4-5 \mu \mathrm{m})$ were serially cut, stained with hematoxylin and eosin and histologically examined. Selected sections were immunostained with anti-p53 antibody (DO-7, Histofine kit; Nichirei, Tokyo, Japan). The association of ESD artifacts with clinical findings was analyzed using the Chi-square test, Fisher's exact test, unpaired t-test and Mann-Whitney $\mathrm{U}$ test. $\mathrm{P}<0.05$ was considered to indicate a statistically significant difference.

\section{Results}

Nuclear artifacts. In 69 ESD specimens (71.1\%), possible nuclear artifacts were scattered in the peripheral portions 

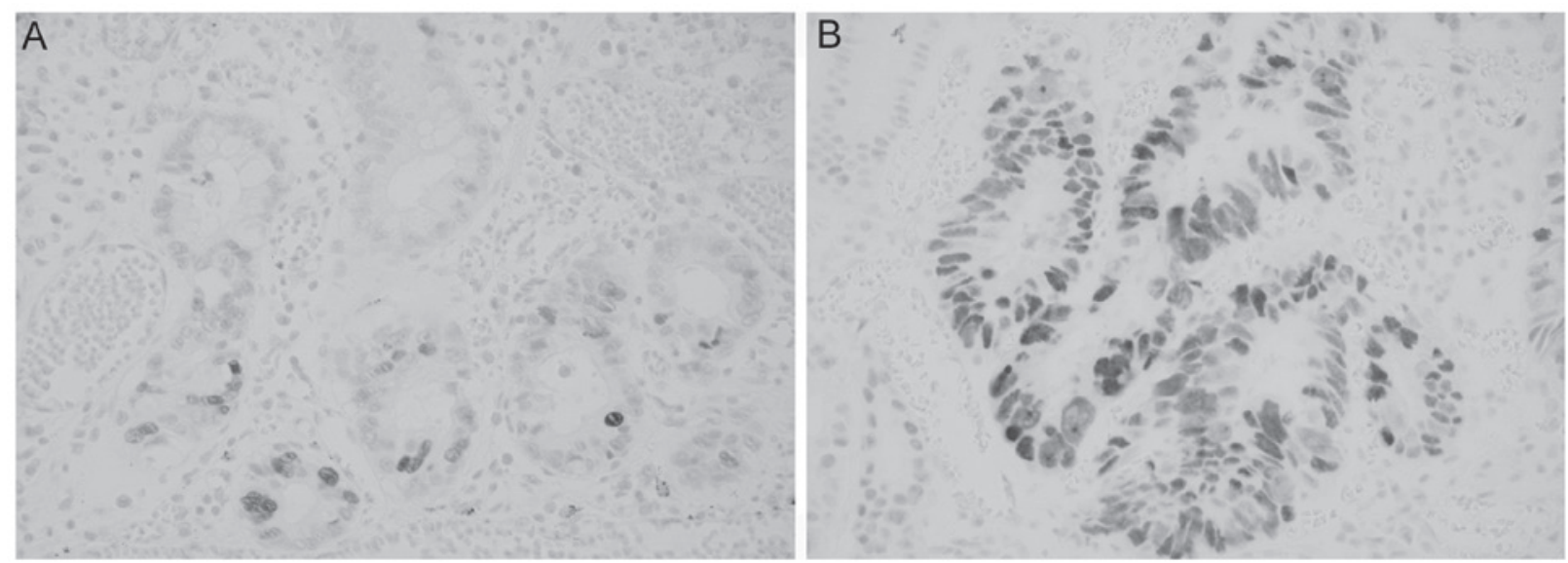

Figure 3. (A) Scattered p53 positivity in endoscopic submucosal dissection (ESD)-related dysplasia-like artifacts and (B) strong and diffuse p53-positive pattern in true adenocarcinoma in the same ESD specimen.

near/beneath preoperative marking-related erosive and/or coagulated lesions and exhibited multinucleated figures (68 specimens, 70.1\%; Fig. 1A-C) and atypical mitotic-like figures (28 specimens, 28.9\%). The former sometimes simulated gigantic nuclei (Fig. 1D) and the latter included a tripolar spindle-like configuration (8 specimens, 8.2\%; Fig. 1E) and/or bizarre asterisk or spindle figures (19 specimens, 19.9\%; Fig. 1F). 'Fresh' and 'previous' marking areas were not distinguished histologically. The patient sources for each ESD specimen were 64 men and 5 women and the age at which each ESD specimen was obtained ranged between 44 and 84 years (mean, 60.9 years). The final diagnoses included 52 cases with EGA, 1 with advanced gastric cancer, 10 tubular adenomas, 4 hyperplastic polyps and 2 carcinoid tumors. The ESD changes were mainly minute and the majority were easily recognizable as artifacts. In 13 cases (13.4\%), however, they mimicked dysplasia, as they were focally aggregated concomitant with cribriform-like structures, nuclear enlargement, nuclear hyperchromatism and/or an irregular nuclear arrangement. In 1 case, the artifacts were initially misdiagnosed as adenocarcinoma (Fig. 2). However, in the deep cut sections, the dysplastic nature tended to disappear and sometimes other degenerative findings became more prominent. In 11 of the 12 cases examined $(91.7 \%)$, scattered nuclear p53 positivity was detected in dysplasia-like artifacts (Fig. 3A). However, this staining pattern was different from diffusely p53-positive adenocarcinoma cells (Fig. 3B), which were found in 6 of the 8 EGAs examined. As a control study of ESD artifacts, we selected 69 age- and gender-matched control cases comprising 64 men and 5 women, with a mean age of 60.9 years (range, 44-84 years) selected from 79 gastrectomy cases without ESD. Similar nuclear artifacts were not identified in these gastrectomy specimens.

Association of ESD artifacts with clinical findings. The association between ESD artifacts and clinical findings was analyzed in each ESD specimen and is summarized in Tables I and II. Multinucleated figures were associated with larger-sized ESD specimens $(\mathrm{P}=0.003)$, presence of previous marking $(\mathrm{P}<0.001)$ and higher frequency of marking $(\mathrm{P}<0.001)$, but not with age at ESD, number of ESD specimens in individual patients, ESD procedure time, previous vs. fresh marking, the device used in fresh marking (APC vs. non-APC), the dissecting device (IT vs. non-IT knife), or diathermic hemostasis. Atypical mitotic-like figures were associated with previous $(\mathrm{P}=0.002)$ as well as fresh marking $(\mathrm{P}=0.007)$, but not with other findings. Dysplasia-like artifacts were associated with older age $(\mathrm{P}=0.031)$, but not with other findings.

Patient outcome. Of the 62 patients with nuclear artifacts, 2 died from other causes ( 1 from pulmonary fibrosis and 1 from heatstroke) $\geq 2$ years after ESD. The remaining 60 patients were alive, with a mean follow-up period of 30.3 months (range, 4-53 months), although 3 underwent additional gastrectomy due to positive submucosal margins ( 2 patients) and multiple carcinoid tumors (1 patient). No patients exhibited recurrent cancerous growth adjacent to the post-ESD sites.

\section{Discussion}

In this study, we observed unique multinucleated and/or atypical mitotic-like figures in $71 \%$ of the ESD specimens. These figures were not found in age- and gender-matched control gastrectomy specimens, suggesting that the artifacts were ESD-related. Regarding ER/ESD-related gastrointestinal changes, previous studies emphasized that the artifacts disturbed histological diagnosis and assessment of marginal status $(1,3,4,9,10,12)$, but did not focus on the nuclear changes. To the best of our knowledge, the present study is the first to describe ESD-related nuclear artifacts in detail. Nuclear artifacts were found close to marking-related erosive and/or depressed coagulation, corresponding to thermal artifacts, also called electrothermal, diathermic, or cautery-related changes (1,3,4,9-11). Therefore, nuclear artifacts themselves would have contributed to the thermal artifacts. By examining 1 section only, the associated marking-related thermal changes may be unclear. However, we believe that further examination of the next adjacent and/or deep cut sections and comparative observation with the gross photographs of the ESD specimens may reveal the distinct marking points close to the nuclear changes.

The multinucleated artifacts partly resembled previously described multinucleated cells in esophageal and colorectal 
Table I. Associations of artifactual nuclear changes in ESD specimens with clinical findings.

\begin{tabular}{|c|c|c|c|c|c|c|}
\hline Variables & $\begin{array}{l}\text { Multinucleated } \\
\text { figures }(+) \\
(n=68)\end{array}$ & $\begin{array}{l}\text { Multinucleated } \\
\text { figures }(-) \\
(n=29)\end{array}$ & P-value & $\begin{array}{c}\text { Atypical } \\
\text { mitotic-like } \\
\text { figures }(+) \\
(n=28)\end{array}$ & $\begin{array}{c}\text { Atypical } \\
\text { mitotic-like } \\
\text { figures (-) } \\
(n=69)\end{array}$ & P-value \\
\hline $\begin{array}{l}\text { Age at ESD }{ }^{\mathrm{a}}, \text { years } \\
\text { Mean (range) }\end{array}$ & $60.9(44-84)$ & $59.5(40-84)$ & 0.779 & $60.4(46-84)$ & $60.6(40-81)$ & 0.954 \\
\hline $\begin{array}{l}\text { Size of ESD specimens, cm } \\
\text { Mean (range) }\end{array}$ & $3.9(1.2-9.0)$ & $3.1(1.4-5.6)$ & 0.003 & $3.4(1.2-5.4)$ & $3.8(1.4-9.0)$ & 0.321 \\
\hline $\begin{array}{l}\text { Procedure time of ESD, min } \\
\text { Mean (range) }\end{array}$ & $94.3(15-470)$ & $85.5(7-250)$ & 0.381 & $75.9(15-470)$ & $97.7(15-470)$ & 0.199 \\
\hline Preoperative marking $+/$ - & $68 / 0$ & $27 / 2$ & 0.159 & $28 / 0$ & $67 / 2$ & 0.729 \\
\hline Previous ${ }^{\mathrm{b}}$ marking $+/-$ & $66 / 2$ & $10 / 19$ & $<0.001$ & $28 / 0$ & $48 / 21$ & 0.002 \\
\hline Fresh $^{\mathrm{c}}$ marking +/- & $50 / 16$ & $26 / 3$ & 0.135 & $17 / 11$ & $59 / 10$ & 0.007 \\
\hline $\begin{array}{l}\text { Used device in fresh marking } \\
\text { APC/non-APC }\end{array}$ & $44 / 6(n=50)$ & $25 / 1(n=26)$ & 0.454 & $15 / 2(n=17)$ & $54 / 5(n=59)$ & 0.950 \\
\hline $\begin{array}{l}\text { Frequency of marking } \\
\text { Mean (range) }\end{array}$ & $1.7(1-2)$ & $1.24(0-2)$ & $<0.001$ & $1.6(1-2)$ & $1.6(1-2)$ & 0.965 \\
\hline $\begin{array}{l}\text { Used electrical knife } \\
\text { IT/non-IT }^{\mathrm{e}}\end{array}$ & $57 / 11$ & $25 / 4$ & 0.992 & $25 / 3$ & $48 / 21$ & 0.075 \\
\hline Diathermic hemostasis $^{\mathrm{f}}+/-$ & $61 / 7$ & $29 / 0$ & 0.172 & $24 / 4$ & $66 / 3$ & 0.200 \\
\hline
\end{tabular}

Table II. Association of dysplasia-like artifacts in ESD specimens with clinical findings.

\begin{tabular}{|c|c|c|c|}
\hline Variables & $\begin{array}{l}\text { Dysplasia-like artifacts }(+) \\
\qquad(\mathrm{n}=13)\end{array}$ & $\begin{array}{l}\text { Dysplasia-like artifacts }(-) \\
\qquad(\mathrm{n}=84)\end{array}$ & P-value \\
\hline Mean age at ESD ${ }^{\mathrm{a}}$, years (range) & $66.9(50-84)$ & $59.5(40-81)$ & 0.031 \\
\hline Mean size of ESD specimens, cm (range) & $4.1(2.5-7.6)$ & $3.6(1.2-9)$ & 0.254 \\
\hline Mean procedure time of ESD, minutes (range) & $89.8(46-204)$ & $91.6(7-470)$ & 0.935 \\
\hline Preoperative marking $+/-$ & $13 / 0$ & $83 / 1$ & 0.280 \\
\hline Previous ${ }^{\mathrm{b}}$ marking $+/-$ & $12 / 1$ & $64 / 20$ & 0.342 \\
\hline Fresh $^{\mathrm{c}}$ marking $+/-$ & $10 / 3$ & $66 / 18$ & 0.820 \\
\hline Used device in fresh marking, APC/non-APC & $9 / 1(n=10)$ & $60 / 6(n=66)$ & 0.621 \\
\hline Mean frequency of marking, (range) & $1.7(1-2)$ & $1.6(0-2)$ & 0.409 \\
\hline Used electrical knife, IT/non-IT ${ }^{\mathrm{e}}$ only & $10 / 3$ & $72 / 12$ & 0.686 \\
\hline Diathermic hemostasis $^{\mathrm{f}}+/-$ & $13 / 0$ & $77 / 7$ & 0.612 \\
\hline
\end{tabular}

${ }^{\mathrm{a} A g e}$ at which ESD was undergone in each ESD specimen. ${ }^{\mathrm{b}} 1-6$ days prior to ESD. ${ }^{\mathrm{c}} 10$ min to a few hours prior to ESD. ${ }^{\mathrm{d} I n c l u d i n g}$ flex and/or precutting knife. ${ }^{e}$ Including flex, hook, flush and/or flex knife. ${ }^{\mathrm{f} U}$ sing Coagrasper ${ }^{\mathrm{TM}}$. ESD, endoscopic submucosal dissection; APC, argon plasma coagulator; IT, insulation-tipped. Bold denotes statistical significance.

biopsy specimens without use of electrothermal devices, which may represent a non-specific, regenerative or degenerative response to injury $(15,16)$. The incidence of such artifacts has been considered rare $(15,16)$, whereas multinucleated artifacts were identified in $70 \%$ of our ESD specimens. These findings suggest that the ESD procedure frequently evokes fundamen- tally rare, degenerative or regenerative multinucleated changes of gastric mucosal cells.

Multinucleated figures were statistically associated with ESD specimen size, frequency of marking and 'previous' marking, but not with 'fresh' marking. Larger ESD specimens may be correlated with a higher frequency of marking. Hence, 
these findings indicate that multinucleated artifacts are closely associated with frequent thermal injury and subsequent degeneration. However, atypical mitotic-like artifacts were associated with 'previous' as well as 'fresh' marking history, but not with the size of ESD specimens or the frequency of marking, denoting the effect of both super-acute destruction and reactive regenerative changes. There may be certain differences in the pathogenesis between these artifacts. Both characteristics were not associated with the number of ESD in the same patient, suggesting that their development may not be associated with the individual vulnerability of each patient.

ESD-related nuclear changes may infrequently mimick dysplasia due to concomitant nuclear enlargement, overlapping, or hyperchromatism and structural abnormality, which have been mentioned in gastric post-EMR biopsy sites (5) and electrical device-related extragastrointestinal specimens $(10,13,14)$. Moreover, in our experience, ESD-related artifacts may be misdiagnosed as adenocarcinoma. Therefore, these artifacts should be distinguished from true neoplasia and dysplasia. Our results suggested the usefulness of further conventional examinations, including histological examination of a deep cut and/or the adjacent section and careful comparison with gross ESD photographs, on the basis of anatomical and histological characteristics of ESD-related artifacts.

The present immunohistochemical study demonstrated a high incidence of p53 positivity in dysplasia-like artifacts ( $92 \%$ of cases examined), although the staining pattern was scattered and differed from the diffuse p53-positive pattern of adenocarcinoma, which was found in $75 \%$ of the EGAs examined. Hibi et al (17) reported scattered p53-positive nuclei in gastric mucosa with persistent Helicobacter pylori infection and suggested that this type of gastritis may induce DNA damage, resulting in nuclear accumulation of wild-type p53 protein. We believe that ESD may induce similar DNA damage, contributing to scattered p53 accumulation in dysplasia-like artifacts.

In conclusion, we described ESD-related multinuclear and atypical mitotic-like artifacts, which, in older patients, may be reminiscent of dysplasia. Further conventional examinations focusing on histological characteristics and favored location of such artifacts may prove useful for discrimination from true dysplasia or cancer.

\section{Acknowledgements}

The authors would like to thank Kenji Okada for excellent technical assistance and Daniel Mrozek for editing the manuscript.

\section{References}

1. Cooper HS, Deppisch LM, Kahn EI, et al: Pathology of the malignant colorectal polyp. Hum Pathol 29: 15-26, 1998.

2. Matsukuma S, Goda K, Sakai Y, Ikegawa K, Morita D, and Kuwabara N: Histopathologic studies of colorectal postendoscopic resection sites: 'skipping electrothermal injury' associated with endoscopic resection procedures. Am J Surg Pathol 23: 459-464, 1999

3. Lauwers GY, Ban S, Mino M, et al: Endoscopic mucosal resection for gastric epithelial neoplasms: a study of 39 cases with emphasis on the evaluation of specimens and recommendations for optimal pathologic analysis. Mod Pathol 17: 2-8, 2004.

4. Hull MJ, Mino-Kenudson M, Nishioka NS, et al: Endoscopic mucosal resection: an improved diagnostic procedure for early gastroesophageal epithelial neoplasms. Am J Surg Pathol 30: 114-118, 2006.

5. Mitsuhashi T, Lauwers GY, Ban S, et al: Post-gastric endoscopic mucosal resection surveillance biopsies: evaluation of mucosal changes and recognition of potential mimics of residual adenocarcinoma. Am J Surg Pathol 30: 650-656, 2006.

6. Kim SG: Endoscopic treatment for early gastric cancer. J Gastric Cancer 11: 146-154, 2011.

7. Ono H: Early gastric cancer: diagnosis, pathology, treatment techniques and treatment outcomes. Eur J Gastroenterol Hepatol 18: 863-866, 2006.

8. Kakushima N, Ono H, Tanaka M, Takizawa K, Yamaguchi Y and Matsubayashi H: Factors related to lateral margin positivity for cancer in gastric specimens of endoscopic submucosal dissection. Dig Endosc 23: 227-232, 2011.

9. Tanabe H, Iwashita A, Haraoka S, et al: Pathological evaluation concerning curability of endoscopic submucosal dissection (ESD) of early gastric cancer including lesions with obscure margins. Stomach Intestine 41: 53-66, 2006 (In Japanese).

10. Fechner RE: The surgical pathology of iatrogenic lesions. In: Principles and Practice of Surgical Pathology, Silverberg SG (ed). Vol 1. Churchill Livingstone, New York, NY, pp77-99, 1988.

11. Goldstein NS, Watts JC, Neill JS, et al: The effect of electrothermal cautery-assisted resection of diminutive colonic polyps on histopathologic diagnosis. Am J Clin Pathol 115: 356-361, 2001.

12. Dahl J and Greenson JK: Colon. In: Histology for Pathologists. Mills SE (ed). 4th edition. Lippincott Williams \& Wilkins, Philadelphia PA, pp673-695, 2012.

13. Gan E, Costello A, Slavin J and Stillwall RG: Pitfalls in the diagnosis of prostate adenocarcinoma from holmium resection of the prostate. Tech Urol 6: 185-188, 2000.

14. Clarke B and McCluggage WG: Iatrogenic lesions and artefacts in gynaecological pathology. J Clin Pathol 62: 104-112, 2009.

15. Singh SP and Odze RD: Multinucleated epithelial giant cell changes in esophagitis: a clinicopathologic study of 14 cases. Am J Surg Pathol 22: 93-99, 1998.

16. Kambham N, Troxell $M$ and Longacre TA: Multinucleated epithelial giant cells in colorectal polyps: a potential mimic of viropathic and/or dysplastic changes. Am J Surg Pathol 29: 912-919, 2005.

17. Hibi K, Mitomi H, Koizumi W, Tanabe S, Saigenji K and Okayasu I: Enhanced cellular proliferation and p53 accumulation in gastric mucosa chronically infected with Helicobacter pylori. Am J Clin Pathol 108: 26-34, 1997. 